\title{
Cervica Expansive Laminoplasty versus Laminectomy with Fusion for Cervical Ossification of the Posterior Longitudinal Ligament (OPLL)
}

\author{
Seung Hwan Baek, Seung Ho Yoo, Jun Jae Shin ${ }^{\bowtie}$, Sun Yoon Chung, Tae Hong Kim \\ Department of Neurosurgery, Sanggye Paik Hospital, Inje University College of Medicine, Seoul, Korea
}

Objective: The purpose was to comparatively assess cervical sagittal alignment and health-related quality of life (HROOL) outcomes, between patients who underwent cervical expansive laminoplasty (EL) and those who underwent cervical laminectomy with fusion (LF) for cervical ossification of the posterior longitudinal ligament (OPLL) in more than three levels. Methods: We retrospectively evaluated consecutive patients with cervical OPLL undergoing posterior cervical decompression from 2013-2015. We analyzed radiological measurements (C2-7 sagittal vertical axis [C2-7 SVA], C0-2 angle, C2-7 lordotic angle, T1 slope, and range of motion [ROM]) and clinical outcomes (visual analog scale, neck disability index, Short Form-36, and Japanese Orthopaedic Association scores), preoperatively and at the last follow-up. Results: There were 84 patients (63 men; 21 women). Mean follow-up period was 27.7 months (range: 24-48.8). Compared with preoperative C2-7 SVA, postoperative C2-7 SVA was significantly increased in patients in the LF $(13.46 \mathrm{~mm})$ and EL $(2.11 \mathrm{~mm})$ groups. Losses of the cervical lordotic angle and ROM were larger in cervical LF patients than in cervical EL patients. The change in the C2-7 SVA was negatively correlated with the change in the C2-7 lordotic angle and positively correlated with changes in the T1 slope and $\mathrm{C} 0-2$ angle. Improvements in health-related quality of life were similar between the two groups, except for patient-reported neck pain. Conclusion: Cervical EL and LF were effective surgical techniques to improve the patient-reported outcomes and HROOL of patients with OPLL in more than three levels. Cervical EL can preserve sagittal alignment and improve clinical results and neck pain, more than cervical LF. Cervical sagittal imbalance after posterior decompression was related to axial neck pain.

Key Words: Cervical decompression surgery; Ossification of posterior longitudinal ligament; Expansive laminoplasty; Fusion laminectomy, Sagittal alignment

$\triangle$ Corresponding Author: Jun Jae Shin, Department of Neurosurgery, Sanggye Paik Hospital, Inje University College of Medicine, Dongil-ro 1342, Nowon-gu, Seoul 01757, Korea. Tel: +82-2-950-1035, Fax: +82-2-950-1040, E-mail: junjaeshin@gmail.com

\section{INTRODUCTION}

Posterior decompression surgeries such as cervical expansive laminoplasty (EL) or laminectomy with fusion (LF) have been widely performed to adequately decompress the spinal cord, although they cannot directly remove ossification of the posterior longitudinal ligament (OPLL) ${ }^{2,7,11)}$. In patients with cervical myelopathy with OPLL in more than three segments, posterior decompression is more attractive than anterior decompression because this procedure can avoid the need for an anterior route or major strut graft-related problems, such as cerebrospinal fluid leakage, pseudoarthrosis, or graft dislodgment ${ }^{26,31)}$. Posterior decompression surgeries were reported to diminish cervical lordosis preoperatively, and postoperative kyphotic deformities have been considered a source of pain and disability ${ }^{15,21,22,29,32)}$. Therefore, it is important to evaluate the correlation between clinical outcomes and cervical sagittal alignment after posterior cervical decompression. There have been several studies about the clinical outcomes of patients who underwent cervical EL to treat cervical spondylotic myelopathy ${ }^{6,13,16,30)}$. However, few studies have evaluated whether posterior decompression techniques such as EL or LF lead to cervical kyphosis and if these procedures could be used to achieve favorable clinical outcomes in patients with multilevel cervical OPLL ${ }^{13)}$.

We compared cervical EL with LF to treat cervical compressive myelopathy (CCM) in patients with OPLL in more than three levels. The purpose of this study was to comparatively assess cervical sagittal alignment and clinical outcomes, including patient-reported pain and quality of life, between patients who underwent cervical EL and those who underwent cervical LF.

\section{MATERIALS AND METHODS}

\section{Patient demographics}

This study was approved by the local ethical committee 
and the institutional review board. This was a retrospective cohort study.

From January 2013 to January 2015, we treated 415 consecutive patients with cervical spine diseases. Of these 415 patients, $99(23.9 \%)$ had ventral cord compression with OPLL in more than three levels. Two patients who underwent anterior cervical decompression and 13 who underwent combined anteroposterior surgeries were excluded from this study.

Eighty-four patients (63 men and 21 women) with a mean age of 59.4 years (range: $38-77$ years) underwent posterior cervical decompression surgery such as cervical EL or LF to treat CCM associated with OPLL in more than three levels. CCM was diagnosed using magnetic resonance imaging (MRI) with radiological confirmation, and the diagnosis was determined when there was one or more upper motor neuron domain (e.g., spasticity, hyper-reflexia, or a positive Babinski sign), based on a neurological examination ${ }^{4)}$.

We excluded patients with one- or two-level segmental OPLL, other acute traumatic cord injuries with cervical bony fractures caused by high-energy trauma, or another confirmed neurological disorder (e.g., cerebral palsy, Parkinson's disease, multiple sclerosis, or polio). Patients with ossification of the yellow ligament and those with a history of cervical spine surgery were also excluded.

Patient age, sex, duration of symptoms, medical conditions, and radiographic findings were investigated. The presence of OPLL was confirmed with X-ray or three-dimensional computed tomography (CT). All patients underwent high-resolution MRI using a $1.5 \mathrm{~T}$ Avanto unit (Siemens Medical System, Erlangen, Germany) imaging unit prior to surgery. We defined complications as events that occurred within 1 month postoperatively. We analyzed neurological recovery in patients who completed at least 24 months of follow-up.

\section{Operative technique}

The patients were placed in the prone position under general anesthesia. A standard posterior midline approach was used. Surgical techniques were selected according to patient factors (age or general condition) and the characteristic of the disease (instability or cord compression ratio).

Cervical EL was selected based on the patient's neurological symptoms and the number of compressed cord levels, including the narrowest side, on images. Unilateral or bilateral gutters were made using a high-speed drill at the area between the facet joints and laminae, and the spinous processes were split sagittally with a high-speed burr. The spinal canal was enlarged by opening the split laminae unilaterally or bilaterally with a spreader. Posterior cervical titanium miniplates (Centerpiece ${ }^{\circledR}$, Medtronic Sofamor Danek, Memphis, TN, USA) or hydroxyapatite spacers (Apacerum ${ }^{\circledR}$, Asahi Optical Co., Ltd., Tokyo, Japan) were used to keep the "door" open.
When we performed cervical LF, we attempted to maintain preoperative cervical lordosis using a posterior screw-rod system for OPLL patients with segmental instability. We prefer to perform cervical LF to widely decompress the spinal cord in older patients and those with severe cord compression caused by OPLL. Following complete decompression of the neural elements, lateral mass or pedicle screw and rod instrumentation $\left(\right.$ Vertex $^{\circledR}$, Medtronic Sofamor Danek, Memphis, TN, USA) was performed. The perizygapophysial joints were filled with autologous bone fragments from the excised lamina. Postoperatively, all patients were required to wear Miami neck collars or Philadelphia braces for approximately 3 months. The patients were followed clinically with plain and dynamic radiography at 1 , 3 , and 6 months and then every 6 months postoperatively.

\section{Radiographic assessment}

Cervical spine radiological measurements were made by assessing the following parameters: (1) C2-7 sagittal vertical axis (C2-7 SVA), (2) C0-2 angle, (3) C2-7 lordotic angle, (4) T1 slope (T1S), and (5) total range of motion (ROM). The C2-7 SVA was defined as the distance from the posterosuperior corner of $\mathrm{C} 7$ and a vertical line from the center of the $\mathrm{C} 2$ body. The $\mathrm{C} 0-2$ angle was measured using a Cobb angle between the McGregor line and $\mathrm{C} 2$ lower end plate. Cervical lordosis was assessed with the $\mathrm{C} 2-7$ lordotic angle. The $\mathrm{C} 2-7$ lordotic angle was defined as the Cobb angle formed by the inferior endplate of $\mathrm{C} 2$ and $\mathrm{C} 7$ on a standing lateral radiograph. The T1S was measured as the angle between a horizontal line and the superior endplate of T1 (Fig. 1). The total ROM of the

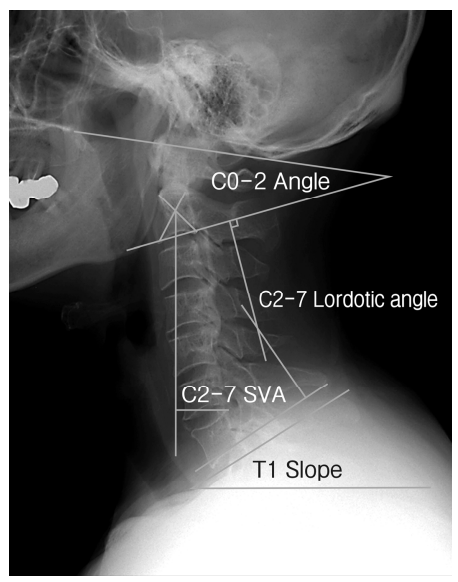

Fig. 1. Radiological parameters. The $\mathrm{C} 2-7$ sagittal vertical axis (the distance from the posterosuperior corner of $\mathrm{C} 7$ and a vertical line from the center of the $\mathrm{C} 2$ body), C0-2 angle (a Cobb angle between the McGregor line and the $\mathrm{C} 2$ lower end plate), C2-7 lordotic angle (Cobb angle between the inferior endplate of $\mathrm{C} 2$ and $\mathrm{C7}$ ), and $\mathrm{T} 1$ slope (the angle between a horizontal line and the superior endplate of T1) were measured on neutral lateral radiographs. 
cervical spine was defined as the summation of alignment during flexion and extension on radiographs. Changes in the cervical alignment were defined as the difference between the pre- and postoperative cervical sagittal parameters ${ }^{9,19)}$.

CT scanning was performed preoperatively for all patients. The occupying ratio of the spinal canal was measured on a CT sagittal view (Fig. 2). We also assessed signal intensity changes on MRI T2-weighted images. Radiographic measurement data were collected by two observers (SHB and SHY).

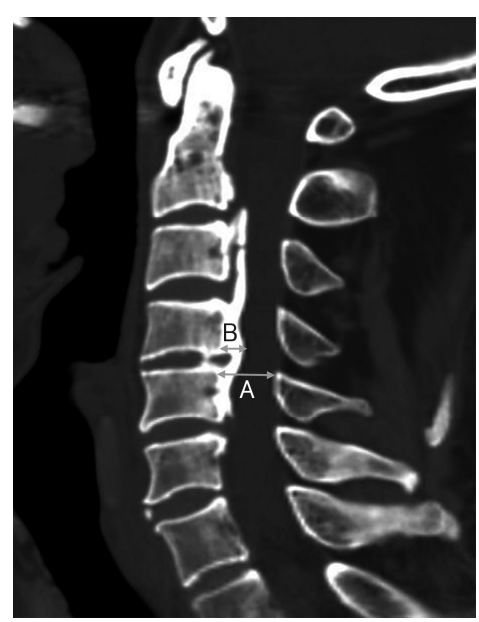

Fig. 2. The occupying ratio of the spinal canal. (A) The anteroposterior diameter of the spinal canal. (B) The maximum anteroposterior thickness of the ossified ligament on a sagittal computed tomography image. The occupying ratio $(\%)$ of the spinal canal was determined according to the formula (B/A)X100.
The data were reviewed by a professor (JJS).

\section{Clinical outcomes assessment}

The clinical outcomes were assessed using the patient-reported visual analog scale for neck pain (VAS-N) and arm pain (VAS-A), neck disability index (NDI), Short Form 36 health survey questionnaire (SF-36), and Japanese Orthopaedic Association (JOA) score. These data were obtained for all patients preoperatively and for at least 2 years postoperatively. The neurological outcomes were evaluated according to the JOA scale for cervical myelopathy ${ }^{5,8)}$. The postoperative recovery rates were calculated using the following formula:

JOA recovery ratio $(\%)=$ (postoperative JOA score-preoperative JOA score)/(17 (full score)-preoperative JOA score)

\section{Statistical analysis}

All values are expressed as the mean \pm standard deviation or percentage. Normally distributed data were compared using Student's t-test or a chi-squared test, as appropriate for the data set. Data with a non-normal distribution were evaluated using a nonparametric analysis, and the Mann-Whitney U test was used to evaluate differences between the two groups. Pearson's correlation analysis was used to evaluate the relationships between the JOA recovery ratio and age, cervical spine parameters, occupying ratio of the spinal canal, and pre- and postoperative VAS-N, VAS-A, NDI, SF-36, and JOA scores. All statistical analyses were performed using MedCalc version 18.5

Table 1. Patient demographics

\begin{tabular}{|c|c|c|c|}
\hline & Laminectomy and fusion $(\mathrm{n}=36)$ & Laminoplasty $(\mathrm{n}=48)$ & $p$-value \\
\hline Age (years) & $65.81 \pm 7.99$ & $54.52 \pm 9.57$ & 0.0001 \\
\hline Sex M:F & $31: 5$ & $32: 16$ & 0.0470 \\
\hline Type of OPLL & & & 0.6946 \\
\hline Continuous & 7 & 11 & \\
\hline Segmental & 14 & 13 & \\
\hline Mixed & 15 & 24 & \\
\hline Preoperative occupying ratio (\%) & $48.77 \pm 12.29$ & $54.50 \pm 14.29$ & 0.0639 \\
\hline Preoperative T2WI MR SI change, no of patients (\%) & $23(63.89)$ & $28(58.33)$ & 0.6125 \\
\hline \multicolumn{4}{|l|}{ Operation level } \\
\hline $\mathrm{C} 3 / 4 / 5 / 6$ & 23 & 46 & 46 \\
\hline $\mathrm{C} 3 / 4 / 5 / 6 / 7$ & 4 & 1 & 1 \\
\hline $\mathrm{C} 2 / 3 / 4 / 5 / 6$ & 7 & 1 & 1 \\
\hline $\mathrm{C} 2 / 3 / 4 / 5 / 6 / 7$ & 2 & 0 & 0 \\
\hline Mean & 3.42 & 3.04 & 0.0001 \\
\hline Operation time (min) & $137.5 \pm 15.71$ & $152.19 \pm 24.56$ & 0.0529 \\
\hline Bleeding amount (mL) & $337.56 \pm 169.73$ & $327.5 \pm 213.99$ & 0.8838 \\
\hline
\end{tabular}

M: male, F: female, OPLL: ossification of posterior longitudinal ligament, T2WI: T2-weighted images, MR: magnetic resonance, SI: signal intensity. All data are expressed as mean \pm SD unless otherwise noted. " $p<0.05$. 

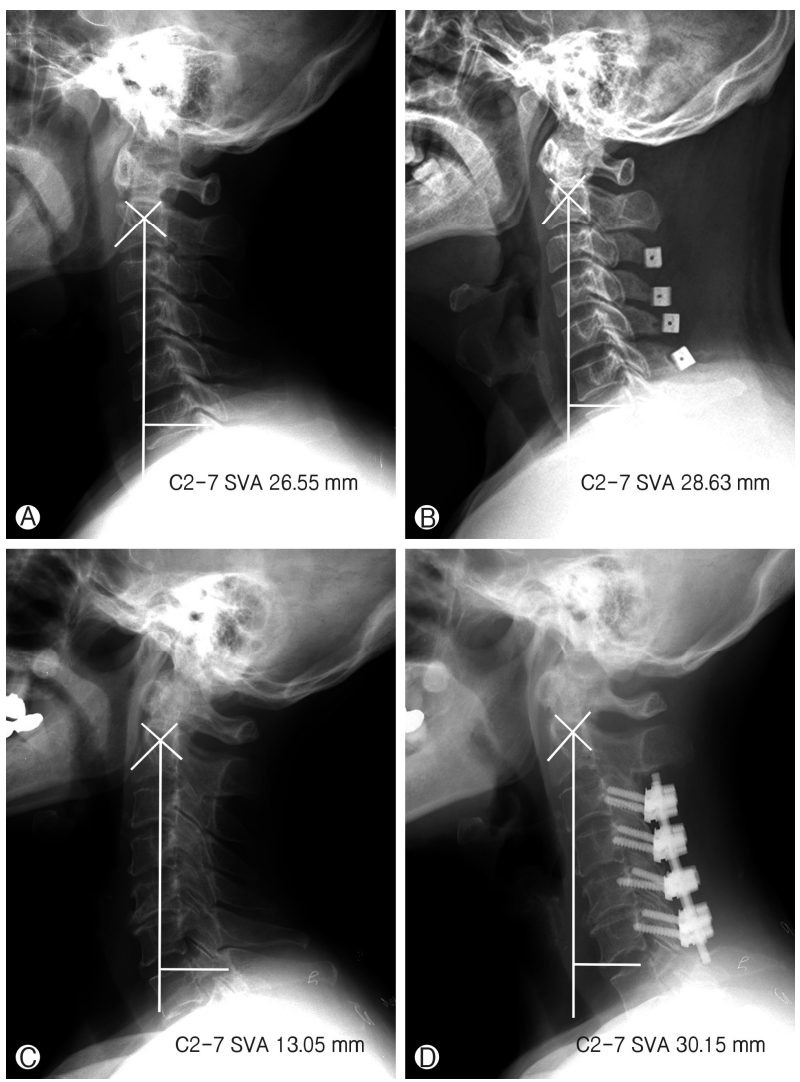

Fig. 3. Comparison of the postoperative C2-7 SVA changes according to the surgical technique. (A) A 49-year-old man had arm numbness and gait disturbance. On preoperative radiographs, C2-7 was $26.55 \mathrm{~mm}$. (B) The patient underwent expansive cervical laminoplasty at C3-4-5-6. At the 2-year follow-up interval, the C2-7 SVA was $28.63 \mathrm{~mm}$. The change in the C2-7 SVA was $2.08 \mathrm{~mm}$. (C) A 65-year-old man had arm weakness, dysesthesia, and an ataxic gait. On preoperative radiographs, the C2-7 SVA was $13.05 \mathrm{~mm}$. (D) The patient underwent cervical laminectomy with fusion at C3-4-5-6. Two years postoperatively, the C2-7 SVA was $30.15 \mathrm{~mm}$. The change in the C2-7 SVA was $17.1 \mathrm{~mm}$. SVA: sagittal vertical axis.

(MedCalc, Mariakerke, Belgium), and a $p$-value $<0.05$ was considered statistically significant.

\section{RESULTS}

Eighty-four patients (63 men and 21 women) were evaluated. The average follow-up period was 27.7 months (range: 24-48.8; 27.32 for cervical LF patients and 28.08 for cervical EL patients). The mean age at the time of surgery was 59.36 years (range: $38-82$ ). The mean ages of the patients in the LF and EL groups were $65.81 \pm 7.99$ and $54.52 \pm 9.57$ years, respectively. The mean age was significantly higher in the LF group than that in the EL group $(p<0.0001)$. There were more male than female patients.

The mixed type of OPLL was the most prevalent in patients who underwent surgery. The average number of operated-upon levels was 3.42 in the LF group and 3.04 in the EL group. OPLL in the upper cervical (C2) or cervicothoracic area (C7) was more frequently observed in LF patients (13/36, 36.11\%) than in EL patients $(2 / 48,4.17 \%)$. The preoperative occupying ratio was higher in the EL group than that in the LF group, while the prevalence of preoperative signal intensity changes on T2-weighted MRIs was higher in the LF group than that in the EF group. However, there was no statistically significant difference between the two groups. There was no significant difference regarding the operation time $(p=0.0529)$ and amount of bleeding ( $p=0.8838$ ) between the two groups. The demographic characteristics of the patients in this study are summarized in Table 1.

\section{Radiological results}

There were no statistically significant differences in the preoperative cervical radiological parameters between the cervical LF and EL groups. After posterior decompression, the C2-7 lordotic angle was reduced, ROM was restricted, and the $\mathrm{C} 0-2$ lordotic angle was increased compared with the preoperative cervical radiological parameters. Postoperatively, cervical alignment in patients in the cervical LF group tilted forward compared with that of patients in the EL group (Fig. 3). The mean C2-7 SVA in the LF group significantly increased from $21.85 \pm 12.32 \mathrm{~mm}$ preoperatively to $35.30 \pm 16.58 \mathrm{~mm}$ at the last follow-up examination, while it increased from $21.33 \pm 10.42 \mathrm{~mm}$ to $23.44 \pm 12.51 \mathrm{~mm}$ in the EL group. The change $(\Delta)$ in the C2-7 SVA was significantly different between the cervical $\mathrm{LF}(13.46 \pm 15.44 \mathrm{~mm})$ and EL $(2.11 \pm 9.13 \mathrm{~mm})$ groups. The mean C2-7 lordotic angle in the LF group significantly decreased from $14.21 \pm 6.29^{\circ}$ preoperatively to $7.48 \pm 10.01^{\circ}$ at the last follow-up examination, while it decreased from $12.53 \pm 7.48^{\circ}$ to $9.78 \pm 8.17^{\circ}$ in the EL group. The change $(\Delta)$ in the $\mathrm{C} 2-7$ lordotic angle was significantly different between the cervical LF $\left(-6.72 \pm 9.93^{\circ}\right)$ and EL groups $\left(-2.75 \pm 6.49^{\circ}\right)$. However, the changes $(\Delta)$ in the $\mathrm{C} 0-2$ angle and T1 slope were not significantly different between the cervical LF and EL groups (Table 2).

The incidence of the loss of cervical lordosis after decompression surgery was $71.43 \%(60 / 84)$, and $75 \%$ of the patients (27/36) in the LF group and $68.75 \%(33 / 48)$ of the patients in the EL group lost cervical lordosis postoperatively. The percentage of patients who lost cervical lordosis postoperatively in the LF group was significantly higher than that of patients in the EL group.

Preoperatively, the average cervical ROMs in patients in the LF and EL groups were $38.37 \pm 12.15^{\circ}$ and $36.26 \pm 15.51^{\circ}$, respectively. At the last follow-up examination, patients in the LF group experienced a significant reduction in ROM compared with those in the EL group (Table 2). The mean reduction of ROM was $27.58 \%$ of the preoperative ROM in the cervical LF group and $60.78 \%$ of the preoperative ROM in the cervical 
EL group. The magnitude of lost ROM was much larger in the cervical LF group than that in the EL group.

The overall change $(\Delta)$ in C2-7 SVA correlated negatively with the change $(\Delta)$ in C2-7 lordotic angle at the final follow-up (R2, 0.0676; $p=0.0169$; Fig. 4A). The change $(\Delta)$ in the $\mathrm{C} 2-7$ SVA demonstrated significantly positive correlations with the

Table 2. Comparison of radiological outcome according to surgical technique

\begin{tabular}{|c|c|c|c|}
\hline & Laminectomy and fusion $(\mathrm{n}=36)$ & Laminoplasty $(\mathrm{n}=48)$ & $p$-value \\
\hline \multicolumn{4}{|l|}{ C2-C7 SVA (mm) } \\
\hline preoperative & $21.85 \pm 12.32$ & $21.33 \pm 10.42$ & 0.7828 \\
\hline postoperative & $35.30 \pm 16.58$ & $23.44 \pm 12.51$ & $0.0003^{*}$ \\
\hline postoperative change $(\Delta)$ & $13.46 \pm 15.44$ & $2.11 \pm 9.13$ & $0.0001^{*}$ \\
\hline \multicolumn{4}{|l|}{ CO-C2 Cobb angle $\left(^{\circ}\right)$} \\
\hline preoperative & $22.27 \pm 9.58$ & $24.35 \pm 9.03$ & 0.1370 \\
\hline postoperative & $25.04 \pm 13.28$ & $26.83 \pm 9.31$ & 0.4924 \\
\hline postoperative change $(\Delta)$ & $2.77 \pm 9.91$ & $2.48 \pm 6.98$ & 0.8743 \\
\hline \multicolumn{4}{|l|}{ C2-C7 Cobb angle $\left(^{\circ}\right)$} \\
\hline preoperative & $14.21 \pm 6.29$ & $12.53 \pm 7.48$ & 0.2813 \\
\hline postoperative & $7.48 \pm 10.01$ & $9.78 \pm 8.17$ & 0.2501 \\
\hline postoperative change $(\Delta)$ & $-6.72 \pm 9.93$ & $-2.75 \pm 6.49$ & $0.0188^{*}$ \\
\hline \multicolumn{4}{|l|}{ T1 slope $\left({ }^{\circ}\right)$} \\
\hline preoperative & $29.50 \pm 5.81$ & $28.05 \pm 6.12$ & 0.2758 \\
\hline postoperative & $26.17 \pm 8.48$ & $25.65 \pm 6.02$ & 0.7564 \\
\hline postoperative change $(\Delta)$ & $-3.33 \pm 8.88$ & $-2.39 \pm 6.04$ & 0.5689 \\
\hline \multicolumn{4}{|l|}{ Total ROM } \\
\hline preoperative & $38.37 \pm 12.15$ & $36.26 \pm 15.51$ & 0.6245 \\
\hline postoperative & $10.58 \pm 21.30$ & $22.04 \pm 15.48$ & $0.0362^{*}$ \\
\hline postoperative change $(\Delta)$ & $-27.79 \pm 12.35$ & $-14.22 \pm 7.68$ & $0.0010^{*}$ \\
\hline
\end{tabular}

C2-7 SVA: C2-7 sagittal vertical axis, ROM: range of motion. All data are expressed as mean \pm SD unless otherwise noted. All data are expressed as mean \pm SD unless otherwise noted. $" \mathrm{p}<0.05$.

Table 3. Comparison of clinical outcome according to surgical technique

\begin{tabular}{|c|c|c|c|}
\hline & Laminectomy and fusion $(n=36)$ & Laminoplasty $(\mathrm{n}=48)$ & $p$-value \\
\hline \multicolumn{4}{|l|}{ VAS score, neck } \\
\hline preoperative & $4.78 \pm 1.91$ & $4.17 \pm 2.31$ & 0.2224 \\
\hline postoperative & $2.78 \pm 1.10$ & $1.83 \pm 1.08$ & $0.0002^{*}$ \\
\hline \multicolumn{4}{|l|}{ VAS score, arm } \\
\hline preoperative & $6.19 \pm 1.80$ & $5.83 \pm 1.62$ & 0.3375 \\
\hline postoperative & $3.83 \pm 1.56$ & $3.35 \pm 1.49$ & 0.1571 \\
\hline \multicolumn{4}{|l|}{ NDI } \\
\hline preoperative & $24.61 \pm 7.63$ & $22.83 \pm 4.74$ & 0.1929 \\
\hline postoperative & $14.19 \pm 6.77$ & $11.44 \pm 4.31$ & 0.0762 \\
\hline \multicolumn{4}{|l|}{ SF-36 } \\
\hline preoperative & $108.67 \pm 9.97$ & $107.75 \pm 8.27$ & 0.2467 \\
\hline postoperative & $96.97 \pm 10.27$ & $96.46 \pm 6.82$ & 0.7838 \\
\hline \multicolumn{4}{|l|}{ JOA score } \\
\hline preoperative & $10.92 \pm 4.09$ & $12.83 \pm 2.27$ & 0.0555 \\
\hline postoperative & $14.78 \pm 1.48$ & $15.29 \pm 1.52$ & 0.1237 \\
\hline JOA recovery ratio $(\%)$ & $53.38 \pm 26.84$ & $59.10 \pm 17.37$ & 0.2400 \\
\hline
\end{tabular}

VAS: visual analog scale, NDI: neck disability index, SF-36: Short Form 36 Health Survey Questionnaire, JOA: Japanese Orthopedic Association score. All data are expressed as mean \pm SD unless otherwise noted. " $p<0.05$. 

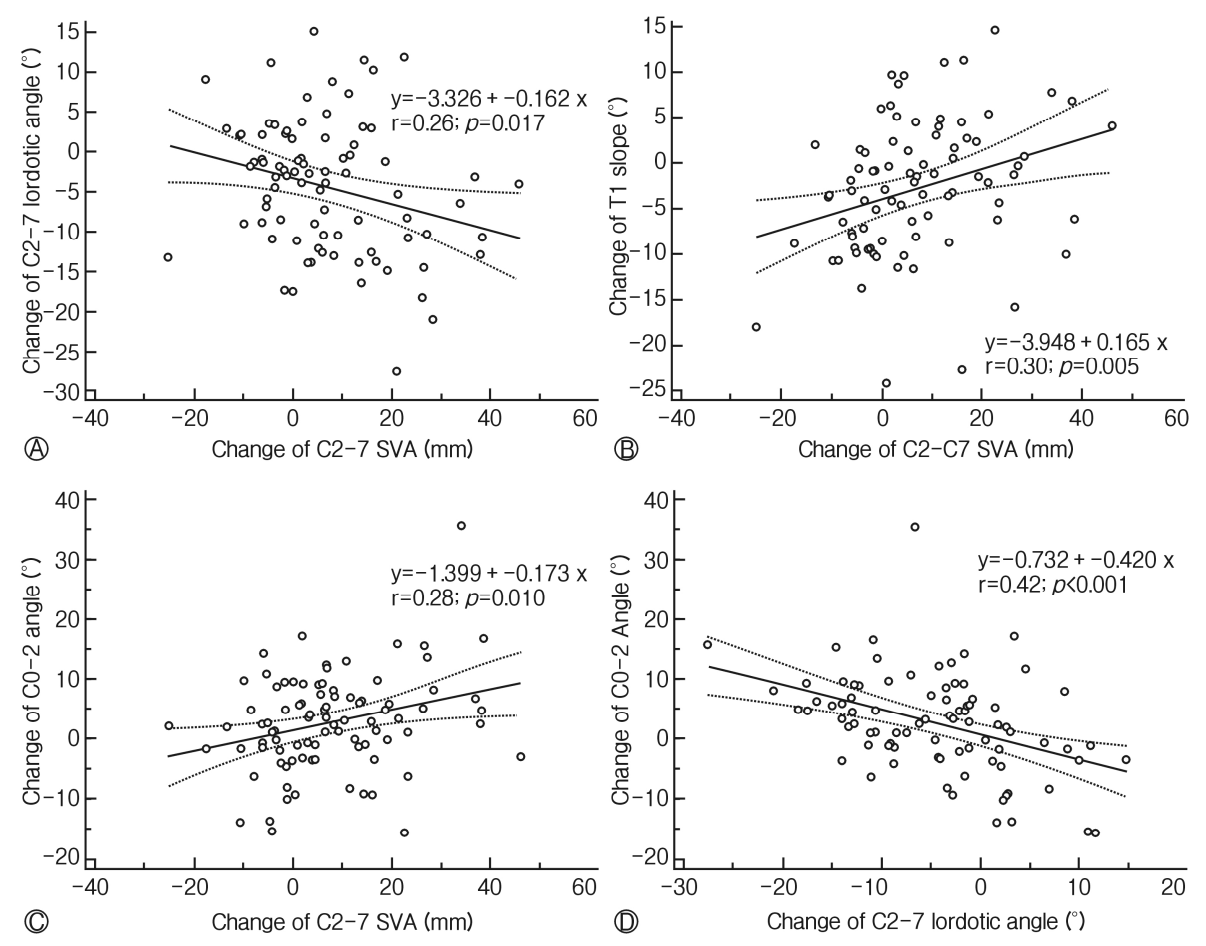

Fig. 4. Relationship of changes in cervical sagittal parameters. (A) Change in C2-7 SVA negatively correlated to the change in C2-7 lordotic angle. (B) Change in C2-7 SVA positively correlated to change in T1 slope. (C) Change in C2-7 SVA significantly correlated to the change in $\mathrm{C} 0-2$ angle. (D) Change in $\mathrm{C} 2-7$ lordotic angle was negatively correlated with the change in the $\mathrm{CO}-2$ angle.

change $(\Delta)$ in the T1S (R2, 0.0907; $p=0.0054$; Fig. 4B) and $\mathrm{C} 0-2$ angle (R2, $0.0776 ; p=0.0 .0103$; Fig. $4 \mathrm{C})$. The change $(\Delta)$ in the $\mathrm{C} 2-7$ lordotic angle was negatively correlated with the change $(\Delta)$ in the $\mathrm{C} 0-2$ angle (R2, 0.1773; $p=0.0001$; Fig. 4D).

\section{Clinical results}

All clinical parameters were significantly improved at least 24 months postoperatively. The clinical outcomes and healthrelated quality of life (HRQOL) were not significantly different between the two groups, except for the VAS-N scores (Table 3). The mean preoperative and postoperative VAS-N scores in the LF group were $4.78 \pm 1.91$ and $2.78 \pm 1.10$, respectively. The mean VAS-N score in the EL group improved from $4.17 \pm 2.31$ to $1.83 \pm 1.08$. The postoperative VAS-N score showed a statistically significant difference between the two groups $(p=0.0002)$. The JOA recovery ratio was improved in the cervical LF $(53.38 \pm$ 26.84\%) and EL groups (59.1 $17.37 \%$ ), but significant differences were not seen (Table 3).

\section{Complications}

Surgery-related complications occurred in 9 patients (10.71\%) with C5 nerve palsy: $6(16.67 \%, 6 / 36)$ in the LF group and
3 in the EL group $(6.25 \%, 3 / 48)$. Most of the patients recovered within 3 months, but one patient had sustained, unilateral C5 nerve palsy. Furthermore, one patient who underwent cervical EL had a hematoma that developed 3 hours postoperatively. We immediately removed the hematoma, but C5 nerve palsy remained on the patient's right side. Superficial wound infections occurred in 5 patients, but no additional surgery was required to remove instrumentation.

\section{DISCUSSION}

We performed this study to comparatively assess the cervical sagittal alignment and clinical outcomes, including patientreported pain and quality of life, of patients who underwent EL and those who underwent LF. Cervical EL and LF were effective surgical techniques to improve the clinical outcomes of patients with OPLL in more than three levels. Improvements in the patient-reported outcomes and HRQOL were nearly the same for the two techniques. Cervical EL can preserve cervical lordosis and improve range of motion and neck pain, more than cervical laminectomy with fusion can. Cervical sagittal imbalance after posterior decompression surgery was related to axial neck pain. In terms of cervical sagittal alignment, preserving the posterior muscular-ligament complex in subaxial 
lesions was more important than preserving this complex in the upper cervical or cervicothoracic junction.

Many studies reported postoperative kyphotic changes in the cervical spine after posterior decompression surgery ${ }^{15,18,29)}$. The loss of cervical lordosis or kyphotic deformity after laminectomy alone was reported to occur in $33 \%$ to $70.7 \%$ of cases $^{21,22)}$. In a long-term follow-up study, progressive kyphosis resulted in late neurological deterioration ${ }^{26)}$. The EL technique was developed to avoid the complications of laminectomy alone, such as post-laminectomy kyphosis and segmental instability through the resected laminae, and to restore the posterior musculo-ligamentous complex ${ }^{5)}$. However, other investigators found a rate $(35 \%)$ of kyphosis deformity following expansive laminoplasty that was comparable to the reported rates of kyphotic deformity following laminectomy alone ${ }^{25)}$. Therefore, for patients with significant preoperative kyphosis or a high risk of developing kyphosis after laminectomy, cervical laminectomy alone or laminoplasty should be avoided because of postoperative kyphotic deformity, and posterior laminectomy with instrumented fusion is indicated ${ }^{12)}$. Some authors insisted that posterior screw augmentation after laminectomy could prevent postoperative kyphotic deformities, axial pain, and instability ${ }^{1,12)}$. Anakwenze et al. reported that post-laminectomy kyphosis might be prevented in patients undergoing posterior screw fusion ${ }^{1)}$.

The current study showed that posterior decompression surgery caused loss of the preoperative cervical lordotic angle. Patients who underwent cervical LF had a higher incidence of postoperative kyphosis and loss of preoperative lordosis than did those who underwent EL.

Sakai et al. reported that the cervical C2-7 SVA increased and the cervical lordotic angle decreased after cervical laminoplasty $^{26}$. In this study, we found that the C2-7 SVA increased after posterior decompression surgery. Particularly, the cervical C2-7 SVA increased more in patients who underwent cervical LF than in those who underwent EL. As a result, supplementation with screw fixation after cervical laminectomy did not allow us to maintain the preoperative cervical alignment. When cervical laminectomy with fusion is performed, the posterior neck muscles should be dissected extensively to confirm the screw's entry point, compared with the muscle exposure procedure used in EL. As the level of surgical invasion of the posterior muscular-ligament complex increases, cervical sagittal balance decreases. Despite the use of posterior screw augmentation to stabilize the bony and joint structures, the compensatory mechanism did not work after the musculo-ligamentous complex was damaged. To reduce damage to the musculo-ligamentous complex, Kim et al. developed myoarchitectonic spinolaminoplasty without damaging the attachments of the semispinalis cervicis, multifidus, interspinalis, and rotator muscles, which could prevent postoperative instability, decrease the incidence of postoperative kyphosis, and preserve the ROM of the C2-7 angle at 1 year after surgery ${ }^{7,27)}$.
Maintaining the paraspinal musculature at $\mathrm{C} 2$ and $\mathrm{C} 7$ is important during a posterior cervical operation because involvement of the $\mathrm{C} 2$ and $\mathrm{C} 7$ vertebrae might affect the postoperative cervical sagittal balance ${ }^{24)}$. We compared original data (in which the $\mathrm{C} 2$ and $\mathrm{C} 7$ level was included) with subtracted data (in which the $\mathrm{C} 2$ and $\mathrm{C} 7$ was not included) to assess the risk of kyphosis. The change $(\Delta)$ in the C2-7 SVA was significantly increased in the cervical LF group compared with that in the EL group. Postoperatively, the LF group had large alterations in the C2-7 lordotic angle compared with the preoperative angle. These results were similar to the original results.

Sagittal imbalance in one region of the spine can influence other regions. Damaged parts of the posterior cervical spine would affect the compensatory mechanism of the upper cervical or subaxial spine ${ }^{17)}$. In the present study, the C2-7 SVA was positively correlated with the $\mathrm{T} 1$ slope and $\mathrm{C} 0-2$ angle and negatively correlated with the $\mathrm{C} 2-7$ lordotic angle. The $\mathrm{C} 2-7$ lordotic angle was negatively correlated with the $\mathrm{C} 0-2$ angle, which is consistent with the results of previous studies ${ }^{17,29)}$. However, compensation was not enough to maintain the preoperative cervical alignment and prevent kyphosis from occurring postoperatively.

\section{Clinical outcome}

Previous studies have addressed the relationship between cervical sagittal alignment and HRQOL ${ }^{10,15,29)}$. Lee et al. reported that the C2-7 SVA was significantly correlated with HRQOL ${ }^{15}$. However, some researchers reported that cervical sagittal imbalance was not related to postoperative clinical outcomes ${ }^{14)}$. We found that decompression surgeries, including cervical LF and EL, were associated with similar improvements in QOL scores such as the NDI, SF-36, and JOA scores. The VAS-N score after decompression was significantly correlated with the C2-7 SVA among various cervical sagittal parameters. We thought that invasion of the posterior muscular-ligament complex, loss of cervical lordosis, and anterior neck tilt increased energy expenditure in relation to the head's position and horizontal gaze. These factors could explain why patients experienced neck pain after undergoing posterior decompression surgery, particularly those who underwent the cervical LF technique. Consequentially, sagittal imbalance after posterior decompression surgery of the cervical spine would be related to the clini cal outcome, particularly axial neck pain.

\section{ROM}

ROM of the cervical spine was reported to be restricted by posterior decompression surgeries such as EL or $\mathrm{LF}^{6,20)}$. Several reports have described reductions in ROM of 30\% to $70 \%$ of the preoperative ROM after cervical laminoplasty ${ }^{6,20)}$. Blizzard et al. reported that the ROM was reduced by up to 
$27.11 \%$ of the preoperative ROM after cervical $\mathrm{LF}^{2)}$. In our study, patients in the cervical LF group experienced a significant reduction in ROM compared with those in the EL group. Cervical laminoplasty is advantageous because it can preserve the cervical spine's motions compared with multilevel LF.

\section{Complications (C5 nerve palsy)}

Shou et al. performed a meta-analysis that focused on the prevalence of C5 nerve root palsy postoperatively and demonstrated that cervical LF was associated with a high prevalence of C5 nerve root palsy $(11.0 \%)^{3,28)}$. The findings of the current study are consistent with those of prior studies ${ }^{23,28)}$ reporting that there was a higher prevalence of C5 nerve root palsy after cervical LF (16.67\%) than there was after EL (6.25\%).

\section{Limitations}

This study has several limitations. First, the study was a retrospective, consecutive patient series, and inequalities between the groups inevitably existed. There is an inherent patient selection bias when comparing surgical procedures because indications for expansive laminoplasty and cervical laminectomy with fusion tend to be different. Second, our radiographical analysis period was relatively short (mean: 27.7 months), and a longer follow-up period will be required to determine the durability of our findings. Third, the whole-spine sagittal alignment, which was affected by cervical alignment postoperatively, was not included in our analysis. Further studies are needed to determine whether patients will have whole-spine sagittal imbalance in the long term.

\section{CONCLUSION}

Cervical EL preserved cervical lordosis and improved ROM and neck pain, more than LF for treatment of more than 3 levels of cervical OPLL. Sagittal imbalance after posterior decompression surgery of the cervical spine was related to the clinical outcome, particularly axial neck pain. We found that the preservation of the posterior musculo-ligamentous complex in subaxial lesions was more important in maintaining the sagittal alignment than the complex in the upper cervical or cervicothoracic junction.

\section{- Abbreviations}

$\mathrm{EL}=$ Expansive laminoplasty; $\mathrm{LF}=$ laminectomy with fusion; OPLL $=$ ossification of the posterior longitudinal ligament; $\mathrm{C} 2-7$ $\mathrm{SVA}=\mathrm{C} 2-7$ sagittal vertical axis; $\mathrm{T} 1 \mathrm{~S}=\mathrm{T} 1$ slope; $\mathrm{ROM}=$ range of motion; VAS=visual analog scale; VAS-N=visual analog scale for the neck; VAS-A=visual analog scale for the arm; NDI=neck disability index; SF-36=Short Form 36 Health Survey Question- naire; JOA=Japanese Orthopedic Association; HRQOL=healthrelated quality of life; $\mathrm{QOL}=$ quality of life; $\mathrm{MRI}=$ magnetic resonance imaging; 3D CT=three-dimensional computed tomography; $\mathrm{SI}=$ signal intensity; $\mathrm{T} 2 \mathrm{WI}=\mathrm{MR}$ T2-weighted image.

\section{- Authors' contributions}

SHB and SHY analyzed the data and drafted the manuscript. JJS and SHB revised the manuscript critically for important intellectual content and approved the final version to be submitted. SHY designed the study and performed the statistical analysis. SYC and THK conceived the study, participated in its design and coordination, and helped to draft the manuscript. All authors have read and approved the final manuscript.

\section{- Acknowledgements}

The authors wish to thank Hyun Kyung Park, $\mathrm{PhD}$, for her contributions in drafting the manuscript and revising it for important intellectual content.

\section{- Ethical approval}

All procedures performed in studies involving human participants were in accordance with the ethical standards of the institutional and/or national research committee and with the 1964 Helsinki declaration and its later amendments or comparable ethical standards.

\section{- Conflicts of interest}

The authors have no financial conflicts of interest.

\section{ORCID}

Jun Jae Shin, https://orcid.org/0000-0002-1503-6343

\section{REFERENCES}

1. Anakwenze OA, Auerbach JD, Buck DW, Garg S, Simon SL, Sutton LN, et al.: The role of concurrent fusion to prevent spinal deformity after intramedullary spinal cord tumor excision in children. J Pediatr Orthop 31:475-479, 2011

2. Blizzard DJ, Caputo AM, Sheets CZ, Klement MR, Michael KW, Isaacs RE, et al.: Laminoplasty versus laminectomy with fusion for the treatment of spondylotic cervical myelopathy: short-term follow-up. Eur Spine J 26:85-93, 2017

3. Chen G, Wang Y, Wang Z, Zhu R, Yang H, Luo Z: Analysis of C5 palsy in cervical myelopathy with massive anterior compression following laminoplasty. Journal of Orthopaedic Surgery and Research 13:26, 2018

4. Cho YE, Shin JJ, Kim KS, Chin DK, Kuh SU, Lee JH, et al.: The relevance of intramedullary high signal intensity and gadolinium (Gd-DTPA) enhancement to the clinical outcome in cervical compressive myelopathy. Eur Spine J 20:2267-2274, 2011

5. Hirabayashi K, Watanabe K, Wakano K, Suzuki N, Satomi K, Ishii Y: Expansive open-door laminoplasty for cervical spinal stenotic myelopathy. Spine (Phila Pa 1976) 8:693-699, 1983 
6. Hyun SJ, Riew KD, Rhim SC: Range of motion loss after cervical laminoplasty: a prospective study with minimum 5-year followup data. Spine J 13:384-390, 2013

7. Kim P, Murata H, Kurokawa R, Takaishi Y, Asakuno K, Kawamoto T: Myoarchitectonic spinolaminoplasty: efficacy in reconstituting the cervical musculature and preserving biomechanical function. J Neurosurg Spine 7:293-304, 2007

8. Kim TH, Ha Y, Shin JJ, Cho YE, Lee JH, Cho WH: Signal intensity ratio on magnetic resonance imaging as a prognostic factor in patients with cervical compressive myelopathy. Medicine (Baltimore) 95:e4649, 2016

9. Kim TH, Lee SY, Kim YC, Park MS, Kim SW: T1 slope as a predictor of kyphotic alignment change after laminoplasty in patients with cervical myelopathy. Spine (Phila Pa 1976) 38:E992997, 2013

10. Kumar VG, Rea GL, Mervis LJ, McGregor JM: Cervical spondylotic myelopathy: functional and radiographic long-term outcome after laminectomy and posterior fusion. Neurosurgery 44 : 771-777; discussion 777-778, 1999

11. Kwon SY, Shin JJ, Lee JH, Cho WH: Prognostic factors for surgical outcome in spinal cord injury associated with ossification of the posterior longitudinal ligament (OPLL). Journal of Orthopaedic Surgery and Research 10:94, 2015

12. Lapsiwala S, Benzel E: Surgical management of cervical myelopathy dealing with the cervical-thoracic junction. The spine journal: official journal of the North American Spine Society 6: 268S-273S, 2006

13. Lee CH, Jahng TA, Hyun SJ, Kim KJ, Kim HJ: Expansive laminoplasty versus laminectomy alone versus laminectomy and fusion for cervical ossification of the posterior longitudinal ligament: Is there a difference in the clinical outcome and sagittal alignment? Clin Spine Surg 29:E9-15, 2016

14. Lee CK, Shin DA, Yi S, Kim KN, Shin HC, Yoon DH, et al.: Correlation between cervical spine sagittal alignment and clinical outcome after cervical laminoplasty for ossification of the posterior longitudinal ligament. J Neurosurg Spine 24:100-107, 2016

15. Lee JS, Youn MS, Shin JK, Goh TS, Kang SS: Relationship between cervical sagittal alignment and quality of life in ankylosing spondylitis. Eur Spine J 24:1199-1203, 2015

16. Lee SE, Chung CK, Jahng TA, Kim HJ: Long-term outcome of laminectomy for cervical ossification of the posterior longitudinal ligament. J Neurosurg Spine 18:465-471, 2013

17. Lee SH, Kim KT, Seo EM, Suk KS, Kwack YH, Son ES: The influence of thoracic inlet alignment on the craniocervical sagittal balance in asymptomatic adults. J Spinal Disord Tech 25:E41-47, 2012

18. Lin S, Zhou F, Sun Y, Chen Z, Zhang F, Pan S: The severity of operative invasion to the posterior muscular-ligament complex influences cervical sagittal balance after open-door laminoplasty. Eur Spine J 24:127-135, 2015

19. Machino M, Yukawa Y, Hida T, Ito K, Nakashima H, Kanbara
S, et al.: Cervical alignment and range of motion after laminoplasty: radiographical data from more than 500 cases with cervical spondylotic myelopathy and a review of the literature. Spine (Phila Pa 1976) 37:E1243-1250, 2012

20. Manzano GR, Casella G, Wang MY, Vanni S, Levi AD: A prospective, randomized trial comparing expansile cervical laminoplasty and cervical laminectomy and fusion for multilevel cervical myelopathy. Neurosurgery 70:264-277, 2012

21. Matsunaga S, Sakou T, Nakanisi K: Analysis of the cervical spine alignment following laminoplasty and laminectomy. Spinal Cord 37:20-24, 1999

22. Mikawa Y, Shikata J, Yamamuro T: Spinal deformity and instability after multilevel cervical laminectomy. Spine (Phila Pa 1976) 12:6-11, 1987

23. Nassr A, Eck JC, Ponnappan RK, Zanoun RR, Donaldson WF, 3rd, Kang JD: The incidence of C5 palsy after multilevel cervical decompression procedures: a review of 750 consecutive cases. Spine (Phila Pa 1976) 37:174-178, 2012

24. Nolan JP, Jr., Sherk HH: Biomechanical evaluation of the extensor musculature of the cervical spine. Spine (Phila Pa 1976) 13:9-11, 1988

25. Ratliff JK, Cooper PR: Cervical laminoplasty: a critical review. J Neurosurg 98:230-238, 2003

26. Sakai K, Yoshii T, Hirai T, Arai Y, Shinomiya K, Okawa A: Impact of the surgical treatment for degenerative cervical myelopathy on the preoperative cervical sagittal balance: a review of prospective comparative cohort between anterior decompression with fusion and laminoplasty. Eur Spine J 26:104-112, 2017

27. Shiraishi T: A new technique for exposure of the cervical spine laminae. Technical note. J Neurosurg 96:122-126, 2002

28. Shou F, Li Z, Wang H, Yan C, Liu Q, Xiao C: Prevalence of C5 nerve root palsy after cervical decompressive surgery: a metaanalysis. Eur Spine J 24:2724-2734, 2015

29. Tang JA, Scheer JK, Smith JS, Deviren V, Bess S, Hart RA, et al.: The impact of standing regional cervical sagittal alignment on outcomes in posterior cervical fusion surgery. Neurosurgery 76 Suppl 1:S14-21; discussion S21, 2015

30. Uchida K, Nakajima H, Sato R, Yayama T, Mwaka ES, Kobayashi $\mathrm{S}$, et al.: Cervical spondylotic myelopathy associated with kyphosis or sagittal sigmoid alignment: outcome after anterior or posterior decompression. Journal of neurosurgery. Spine 11:521528, 2009

31. Wada E, Suzuki S, Kanazawa A, Matsuoka T, Miyamoto S, Yonenobu K: Subtotal corpectomy versus laminoplasty for multilevel cervical spondylotic myelopathy: a long-term follow-up study over 10 years. Spine (Phila Pa 1976) 26:1443-1447; discussion 1448, 2001

32. Zhang JT, Li JQ, Niu RJ, Liu Z, Tong T, Shen Y: Predictors of cervical lordosis loss after laminoplasty in patients with cervical spondylotic myelopathy. Eur Spine J 26:1205-1210, 2017 